\title{
Juego y Simulación de Programas Concurso de Televisión como Técnica Didáctica para Mejorar el Aprendizaje del Vocabulario Inglés en Estudiantes de Habla Hispana
}

Angel B. Mogrovejo ${ }^{1,2 \star}$, Grover Mamani ${ }^{1}$ y Mari L. Tipo ${ }^{1}$

(1) Universidad Nacional de San Agustín de Arequipa, Facultad de Ciencias de la Educación, Arequipa, Perú. (e-mail: amogrovejof@unsa.edu.pe; gmamanil@unsa.edu.pe; mtipo@unsa.edu.pe)

(2) Concytec - Cienciactiva - UNSA, calle Santa Catalina № 117, Arequipa, Perú.

* Autor a quien debe ser dirigida la correspondencia

Recibido Feb. 6, 2018; Aceptado Abr. 2, 2018; Versión final Jun. 29, 2018, Publicado Feb. 2019

\section{Resumen}

Se presentan y discuten los avances y resultados de una investigación sobre la aplicación de la Técnica Didáctica de Juego y Simulación de Programas Concurso de Televisión en el proceso de enseñanza aprendizaje del idioma inglés como segunda lengua, simulando los quiz show y gamificando el aprendizaje. El objetivo es lograr aprendizajes significativos del vocabulario inglés en estudiantes de educación secundaria de habla hispana. La metodología de investigación se basa en comparar el nivel de aprendizaje de los estudiantes entre la pre-prueba y post-prueba, luego de aplicar el juego y la simulación de programas concurso de televisión en diez sesiones de aprendizaje continuas, logrando un entorno diferente al aula tradicional, con información, comunicación y entretenimiento permanente, con actividades de enseñanza muy intensas, con autonomía y altos niveles de motivación. El resultado final demuestra que el aprendizaje del vocabulario inglés mejoró notablemente, desde la pre-prueba en la que obtuvieron un promedio de $11,1 \%$ hasta la post- prueba en la que alcanzaron un promedio de $99.1 \%$ de estudiantes con calificación aprobatoria.

\section{Game and Simulation of Television Contest Programs as a Didactic Technique to Improve the Learning of English Vocabulary in Spanish-Speaking Students}

\begin{abstract}
This paper presents and discusses the application of a Didactic Technique Using Games and Simulations of Television competitions to facilitate the teaching and learning of English, simulating the quiz show and gamifying the learning. The objective to achieve significant learnings in Spanish-speaking high school students to increase their English vocabulary. The research methodology was based on testing students before and after using games and simulations of television competitions in the class, in ten continuous learning sessions. Students had a high level of motivation to learn with information, communication, constant entertainment in class, with a great deal of activities, teamwork learning, constant interaction, which is different to traditional methods. The final result showed that the student's ability to learn English vocabulary improved significantly. Students scored $11.1 \%$ on average during the pre-test and achieved $99.1 \%$ in the average after learning through games and simulations of television contests.
\end{abstract}




\section{INTRODUCCIÓN}

El problema de la enseñanza y del aprendizaje del idioma inglés en estudiantes de habla hispana se debe principalmente a la rutina monótona de la repetición y memorización, generando rechazo y desinterés por aprender el idioma inglés, incluso con la aplicación de nuevas tecnologías. La enseñanza sigue tradicional, la repetición de audios, el uso de libros y guías de estudio, son técnicas e instrumentos anodinos para la enseñanza de la lengua oral, basados en la traducción o el estructuralismo y en las destrezas básicas. La enseñanza de idiomas ha evolucionado desde la enseñanza oral, el enfoque por proyectos, la enseñanza de lenguas asistida por ordenador (ELAO), y el flexible learning model de los últimos tiempos. En este artículo se presentan los avances y resultados del grupo de investigación de la Universidad Nacional de San Agustín, sobre la base de la tesis ganadora de una subvención de fondos concursables del Canon Minero, patrocinada por Concytec (Consejo Nacional de Ciencia y Tecnología e Innovación Tecnológica) a través del convenio Cienciactiva - UNSA, luego de aplicar la Técnica Didáctica de Juego y Simulación de Programas Concurso de Televisión JSPCT, demostrando que ha mejorado notablemente el aprendizaje del vocabulario inglés en estudiantes de educación secundaria de habla hispana, luego de aplicar diez (10) sesiones de aprendizaje en dos Instituciones Educativas: Daniel Goleman de la ciudad de Juliaca y Jens Knudsen de la ciudad de Arequipa, en diez semanas consecutivas en cada institución educativa, con temas básicos que todo estudiante de una segunda lengua L2 debe tener en su vocabulario.

El estudio se inició con un diagnóstico sobre el proceso de enseñanza aprendizaje del idioma inglés como lengua extranjera. Se comprobó que el aprendizaje del idioma inglés continúa siendo tradicional y bastante precario, principalmente porque los profesores a cargo de la enseñanza del idioma inglés pertenecen a otras áreas curriculares y no hablan inglés (según estadísticas nacionales el $70 \%$ de profesores no son del área de ingles). Luego se realizó la evaluación de entrada o pre-test y el resultado fue $11,1 \%$ de aprobados en promedio. Se analizaron diversas técnicas didácticas de mayor motivación en los estudiantes y se eligió la simulación y el juego de los programas concurso de la televisión, quiz shows, gamificando el proceso de enseñanza aprendizaje. Se seleccionaron cinco programas de televisión de gran audiencia, los cuales fueron desarrollados como sesiones de enseñanza aprendizaje del vocabulario inglés, se organizó la simulación en dos sesiones por cada programa haciendo un total de diez sesiones en cada institución educativa, con una sesión por semana de acuerdo a la programación curricular. Se identificaron a los actores, tanto al conductor (el profesor) como a los participantes (los estudiantes), se explicó la secuencia didáctica y se ejecutaron las sesiones de aprendizaje. La motivación para actuar fue intensa y los resultados evidentes en tres de las cuatro habilidades para el aprendizaje de lenguas extranjeras, por considerar que el aprendizaje del vocabulario no incluye el Reading.

Luego de la ejecución de 20 sesiones de aprendizaje en ambas instituciones educativas, los resultados fueron extraordinarios. Se incrementó el porcentaje de estudiantes aprobados en $88 \%$ en los diferentes niveles de calificación, desde el pre test con $11,1 \%$ al post test con $99.1 \%$, en los que solo desaprueba la calificación "C", de acuerdo al sistema de evaluación de Perú. El resultado final muestra que el aprendizaje del vocabulario inglés en estudiantes de habla hispana logró en AD (destacado) 69,44\%; en A (bueno) 27,78\%; en B (regular) $2,77 \%$ y en C (bajo) 0,0\%. El cambio de metodología didáctica, de una tradicional, repetitiva y memorística a una cooperativa y dinámica, gamificando el proceso de enseñanza aprendizaje del vocabulario del idioma ingles logró estos resultados. Para los estudiantes, la enseñanza del inglés es aburrido, monótono y difícil de aprender. El filólogo y traductor Javier Marías, en un artículo publicado en mayo del 2015 hace una crítica devastadora sobre la enseñanza del idioma inglés en España, muy similar al de nuestro medio. Considera como "una de las mayores locuras del sistema educativo español", se basa principalmente en el bajo conocimiento de inglés que tienen los profesores de inglés, y concluye que: "El resultado es un desastre total (ni enseñanza ni bilingüe). Los chicos salen sin saber nada de inglés y aún menos de ciencias o de las asignaturas que hayan caído bajo el dominio del presunto o falso inglés" (Marías, 2015). Para superar este inconveniente, el equipo de investigación se propuso indagar una técnica didáctica que supere el problema del aprendizaje del idioma inglés en estudiantes de habla hispana, evitando la rutina, la simple repetición y memorización, generando como propuesta la Técnica Didáctica de Juego y Simulación de Programas Concurso de Televisión JSPCT (en inglés GSQS), game shows - quiz show, como factor de alta motivación, de interacción permanente y divertida, con el propósito de alcanzar aprendizajes significativos del vocabulario inglés.

El juego desempeña una función importante en el desarrollo de nuestro aprendizaje, tanto en la infancia como en nuestra edad adulta (Foncubierta, 2015). Se puede decir que los juegos comparten un patrón similar, que el aprendizaje tiene lugar en un sistema interpersonal y, por tanto, a través de las interacciones con el docente y con los compañeros del aula, el estudiante aprende los instrumentos cognitivos y comunicativos de su cultura, Ruiz, M. R., \& García, E. G. M. (2005). Uno de los factores que ha hecho que los docentes de lenguas extranjeras tiendan a basar su enseñanza en el enfoque socio-cultural, es el énfasis que este pone en la mediación en el proceso de aprendizaje. De acuerdo a Vygotsky, es a través de la mediación social que el 
conocimiento se hace viable y gana coherencia. La mediación constituye entonces un mecanismo mediante el cual las actividades socio-culturales externas se transforman en funcionamiento mental interno. El objetivo de la teoría de Vygotsky es descubrir y estimular la zona de desarrollo potencial o zona de desarrollo próximo en cada estudiante; en esta teoría se destaca la idea de que el sujeto no se limita a responder a los estímulos de modo pasivo o mecánico, sino que actúa sobre ellos.

El aprendizaje y la enseñanza de lenguas extranjeras se han sometido a un importante cambio de paradigma como resultado de la investigación y las experiencias que han expandido el conocimiento científico y teórico basado en cómo los estudiantes aprenden y adquieren un idioma extranjero. Tradicionalmente, se pensaba que aprender un idioma extranjero era una actividad 'mimética', un proceso que involucró a los estudiantes repitiendo o imitando nueva información. Basado en teorías conductistas de aprendizaje y lingüística estructural, la calidad y la cantidad del lenguaje y la retroalimentación fueron considerados como los principales factores determinantes del éxito del aprendizaje de idiomas (Moeller y Catalano, 2015). Por otro lado, la función principal del profesor ha variado en los últimos tiempos, es necesario un cambio de paradigmas, de técnicas de enseñanza - aprendizaje. El uso de técnicas de aprendizaje activo ayuda a que el profesor deje de ser un sabio en el escenario y pase a ser un guía. El uso del juego en el aula es consistente con los supuestos subyacentes del aprendizaje activo que presenta a los estudiantes como activamente involucrados en su aprendizaje y en su mundo. Esta sencilla herramienta puede ayudar a facilitar el aprendizaje que se basa en el conocimiento cognitivo superior como habilidades que están involucradas con el análisis, síntesis y evaluación de los contenidos. El uso de juegos es particularmente efectivo si el compromiso intelectual es alto y si los estudiantes están más motivados para aprender. Si podemos aprender de una manera divertida e interesante, entonces todos ganamos.

Puede que no sean millones de dólares, pero en educación, representa el premio gordo (Sarason y Banbury 2004). La motivación y el aprendizaje cooperativo son muy importantes en el juego y la simulación, en el que interactúan intensamente el profesor y los estudiantes, experimentan con hechos reales participando activamente y en equipo, en un ambiente de camaradería horizontal. El poder de la simulación y juego reside en la realidad de la práctica comunicativa en la que están envueltos los aprendices, del análisis de la situación a la que se enfrentan y de su toma de decisiones. Sea como fuere, la simulación permite a nuestros estudiantes experimentar con la realidad sin nervios innecesarios, participando activamente en tareas previas, posteriores y aquéllas requeridas durante la simulación propiamente dicha; les entrena a trabajar en equipo de manera cooperativa a la vez que practican y, por lo tanto, mejoran su habilidad con la lengua objeto de estudio (Andreu, 2005:2) citado por (Lorente y Pizarro, 2010). La Técnica Didáctica de Juego y Simulación de Programas Concurso de Televisión JSPCT, mejoró la interacción conflictiva entre profesores y estudiantes, con resultados muy similares a la enseñanza con sistemas tutoriales, Huapaya, C.R., Arona, G.M., \& Lizarralde, F.A. (2005), en la enseñanza de la ingeniería, claramente se perfilan, el estilo de enseñanza de los profesores, el estilo de aprendizaje de los estudiantes y una interacción conflictiva entre ellos. Felder y Brent (2005) sostienen que aquellos instructores que mejor entiendan las diferencias, tendrán la mejor oportunidad de conocer las diversas necesidades de aprendizaje de todos sus estudiantes. El estilo de enseñanza dominante en muchas de nuestras universidades es el experto/autoridad formal, el cual no discrimina preferencias de aprendizaje.

Una de las necesidades de aprendizaje de nuestros estudiantes es la interacción conflictiva entre ellos a través de los medios de comunicación como la televisión, como indica Yassaei, S. (2012) al tratar sobre los medios en el aula de idiomas. Una forma bien conocida de crear un contexto significativo para enseñar inglés es mediante el uso de medios, que puede ser entregado a través de una amplia variedad de impresos, audio y formatos visuales. El desarrollo de la información requiere que los maestros deben estar familiarizados con los medios y la alfabetización mediática. Thoman (2003) argumenta que la alfabetización mediática tiene un papel influyente en los programas educativos, incluido el aprendizaje de un segundo idioma. Los medios se pueden integrar en clases de idiomas en una variedad de formas mediante el desarrollo de actividades basadas en programas de radio, programas de televisión, periódicos y videos. Rucynski (2011) integra la televisión en inglés para la instrucción de una segunda lengua extranjera o instrucción de lenguaje (ESL/EFL) demostrando cómo una variación de las clases de inglés, enseñar con Los Simpson por ejemplo; una famosa serie de TV animada estadounidense con más de 400 episodios.

Finalmente, pretendemos con esta propuesta establecer contacto con la realidad, la enseñanza del idioma ingles en Perú es sumamente preocupante y son necesarios aportes técnicos y científicos al complejo proceso de enseñanza aprendizaje de una segunda lengua. La Técnica Didáctica de Juego y Simulación de Programas Concurso de Televisión, mejora notablemente el proceso de enseñanza aprendizaje del idioma inglés como segunda lengua, una técnica dinámica basada en la enseñanza de lenguas extranjeras sustentadas en el enfoque socio-cultural, en la mediación del proceso de aprendizaje como propone Vygotsky, tratando de alcanzar el mérito académico al publicar los resultados con el propósito de mostrar nuestros avances, como indica Valderrama, (2004): La publicación de artículos en revistas surge, por lo tanto, de la necesidad de 
mostrar los avances logrados en una investigación, para que lleguen a la comunidad especializada en forma eficiente. La satisfacción de esta necesidad es una obligación que debe contraer todo investigador que recibe dinero para sus trabajos de parte de universidades, de gobiernos y de entidades internacionales. Algunas universidades e instituciones gubernamentales de diversos países han comprendido este concepto dual de necesidad-obligación y requieren, por lo tanto, que toda investigación financiada termine con una o más publicaciones (Valderrama, 2001).

\section{METODOLOGÍA}

El equipo de investigación inicia la metodología de investigación con un diagnóstico de preferencias de los estudiantes respecto de los juegos de televisión, programas de concurso de conocimientos game shows quiz show, sobre los siguientes indicadores:

1) Medios auditivos, visuales y audiovisuales; 2) Frecuencia de consumo de televisión; 3) Tipos de programa de televisión; 4) Tiempo de dedicación a la televisión; 5) Preferencias televisivas; y 6) Preferencias de aprendizaje. Luego se aplicó la prueba de entrada para conocer el nivel de aprendizaje del vocabulario del idioma inglés como segunda lengua a nivel de Listening, Writing y Speaking en estudiantes de habla hispana, con la metodología didáctica regularmente aplicada en el aula, considerando un baremo con nota aprobatoria de 11 a 20 puntos (letras $A D, A$ y B), en el que desaprueban solamente quienes necesitan refuerzo de 0 a 10 puntos (letra C), sobre una muestra de 36 estudiantes del cuarto grado de educación secundaria de dos Instituciones Educativas: Daniel Goleman de la ciudad de Juliaca IE1 (16 estudiantes) y Jens Knudsen de la ciudad de Arequipa IE2 (20 estudiantes), considerando la homogeneidad de la muestra en ambos grupos, con similares características de edad, situación social y económica, y el español como principal idioma materno, los que fueron evaluados a través de un mismo examen escrito como pre test y post test y la ficha de observación, luego de 10 semanas, con los indicadores que se muestran en la Tabla 1, para medir y valorar las capacidades auditiva, de escritura y de pronunciación del vocabulario del idioma inglés como segunda lengua.

Tabla 1: Indicadores de evaluación del vocabulario inglés (Listening, Writing y Speaking)

\begin{tabular}{|l|l|l|}
\hline Denominación & \multicolumn{1}{|c|}{ Indicadores de evaluación } & Preguntas \\
\hline Listening & Capacidad auditiva & 1 y 2 \\
\hline Writing & Capacidad para la escritura & 3,4 y 5 \\
\hline Speaking & Capacidad para la pronunciación & 6 y 7 \\
\hline
\end{tabular}

Luego de ejecutar las diez (10) sesiones de aprendizaje con la Técnica Didáctica de Juego y Simulación de Programas Concurso de Televisión JSPCT, se aplicó la prueba de salida para conocer el nivel de aprendizaje del vocabulario del idioma inglés como segunda lengua, valorando los efectos de la técnica didáctica propuesta en el proceso de enseñanza aprendizaje de una segunda lengua. Saber una palabra incluye distintos tipos de conocimiento (Oster, 2009). ¿En qué Consiste "Saber una Palabra"? El conocimiento de una palabra incluye distintos aspectos debido a que cada unidad léxica abarca varios componentes. Por una parte, está el conocimiento semántico (el significado propiamente dicho de la palabra, incluidas las connotaciones y asociaciones que esta conlleva) y por otra parte el conocimiento sobre la forma, que a su vez incluye los aspectos fonéticos y gráficos, así como la composición de la unidad léxica de unidades más pequeñas. El tercer tipo de conocimiento sobre la unidad léxica -aquel relacionado con su uso-comprende las funciones gramaticales de la misma, sus colocaciones (las posibilidades de combinación con otras unidades léxicas) así como las limitaciones de su uso (vid. Nation 2005: 47).

\section{Baremo de evaluación}

Para evaluar el nivel de aprendizaje del vocabulario inglés se utilizó el baremo de evaluación siguiente: 1) Destacado "AD" (Muy bueno, de 18 a 20 puntos); 2) Logrado "A" (Bueno, de 15 a 17 puntos); 3) En proceso "B" (Regular, de 11 a 14 puntos; y 4) Necesita refuerzo "C" (Bajo, de 0 a 10 puntos). Esta escala valora los criterios de Listening, Writing y Speaking, con pruebas o test de evaluación que recogieron información, valorando la competencia lingüística o comunicativa de los estudiantes, su dominio del vocabulario inglés, la capacidad de comunicarse eficazmente, además de contar con la adecuada competencia gramatical, demostrando que saben usar el lenguaje con los miembros de una comunidad lingüística diferente a su lengua materna, es decir, la competencia comunicativa que implica tanto el conocimiento de la lengua inglesa como la habilidad para usarla en situaciones reales. La evaluación del pre-test, sobre la técnica o método audiolingüístico que utiliza recursos tecnológicos como el laboratorio de idiomas, el proyector de diapositivas, las grabadoras portátiles, la práctica de diálogos cortos y repeticiones de frases, asume que la adquisición de una lengua es un proceso memorístico, sin saber o entender su significado, mientras que para el pos-test se 
evaluó el desarrollo de la Técnica Didáctica de Juego y Simulación de Programas Concurso de Televisión JSPCT, eligiendo los programas de televisión más populares como se muestra en la Tabla 2, aplicadas en dos sesiones de aprendizaje cada uno.

Tabla 2: Los quiz shows de televisión más populares

\begin{tabular}{|c|c|c|c|c|}
\hline Objetivo & Niveles & Respuestas & Condiciones & Termina \\
\hline \multicolumn{5}{|c|}{ Programa: Quién Quiere Ser Millonario ((Who wants to be a millonaire) } \\
\hline $\begin{array}{l}\text { Responder } \\
\text { correctamente a } 15 \\
\text { preguntas }\end{array}$ & $\begin{array}{l}3 \text { niveles: de } \\
\text { conocimiento, } \\
\text { general y de } \\
\text { dificultad creciente. }\end{array}$ & $\begin{array}{l}\text { Alternativas: A, B, C y } \\
\text { D }\end{array}$ & $\begin{array}{l}\text { Cada } 5 \text { pregunta } \\
\text { respondida de manera } \\
\text { correcta alcanza un } \\
\text { nivel. Los puntos son } \\
\text { acumulativos. }\end{array}$ & $\begin{array}{l}\text { El concursante contesta } \\
\text { incorrectamente, decide } \\
\text { no responder a una } \\
\text { pregunta, o responde a } \\
\text { todas las preguntas } \\
\text { correctamente. }\end{array}$ \\
\hline \multicolumn{5}{|c|}{ Programa: Password (La palabra secreta) } \\
\hline $\begin{array}{l}\text { Adivinar palabras } \\
\text { secretas, } \\
\text { passwords. }\end{array}$ & $\begin{array}{l}\text { Se juega en pareja } \\
\text { y sólo uno de los } \\
\text { miembros de la } \\
\text { pareja conoce las } \\
\text { palabras. }\end{array}$ & $\begin{array}{l}\text { Solo una respuesta } \\
\text { correcta. }\end{array}$ & $\begin{array}{l}\text { En un tiempo de } 30 \\
\text { segundos, uno le da } \\
\text { una pista al otro con } \\
\text { una palabra diferente. }\end{array}$ & $\begin{array}{l}\text { El concursante que } \\
\text { acierte más passwords } \\
\text { en } 4 \text { rondas pasará a la } \\
\text { ronda final. Si llega al } \\
\text { sexto nivel recibe el } \\
\text { mayor premio. }\end{array}$ \\
\hline \multicolumn{5}{|c|}{ Programa: EI Valor De La Verdad (Nothing but the truth) } \\
\hline $\begin{array}{l}\text { Responder con la } \\
\text { verdad un total de } \\
21 \text { preguntas. }\end{array}$ & $\begin{array}{l}\text { Son } 06 \text { niveles y } \\
\text { cada nivel } \\
\text { incrementa el } \\
\text { monto de dinero. }\end{array}$ & \begin{tabular}{|l|} 
Solo valen \\
respuestas \\
verdaderas, se utiliza \\
el polígrafo como \\
evaluador. \\
\end{tabular} & $\begin{array}{l}\text { Las preguntas } \\
\text { incrementan el monto } \\
\text { de dinero y el grado de } \\
\text { dificultad en cada nivel. }\end{array}$ & $\begin{array}{l}\text { Si el participante no } \\
\text { responde con la verdad } \\
\text { a cualquier pregunta, } \\
\text { pierde todos los puntos } \\
\text { y termina. }\end{array}$ \\
\hline \multicolumn{5}{|c|}{ Programa: Cien Peruanos Dicen (Family Feud) } \\
\hline $\begin{array}{l}\text { Coincidir con las } \\
\text { respuestas de una } \\
\text { encuesta a } 100 \\
\text { peruanos. }\end{array}$ & $\begin{array}{l}\text { Son } 3 \text { niveles o } \\
\text { secuencias. Se } \\
\text { colocan en pantalla } \\
\text { las } 5 \text { respuestas } \\
\text { más populares que } \\
\text { cada grupo tendrá } \\
\text { que acertar. }\end{array}$ & \begin{tabular}{|l|}
5 alternativas de \\
respuestas top de los \\
100 peruanos que \\
aparecen en el \\
tablero y que tienen \\
que coincidir con las \\
respuestas que dé el \\
grupo
\end{tabular} & $\begin{array}{l}\text { La meta es coincidir con } \\
\text { las respuestas de la } \\
\text { encuesta. Se } \\
\text { incrementa con el } \\
\text { segmento "Dinero } \\
\text { rápido": Dos } \\
\text { participantes responden } \\
\text { a las mismas preguntas } \\
\text { en } 20 \text { segundos. } \\
\end{array}$ & $\begin{array}{l}\text { El equipo que alcanza } \\
\text { los } 200 \text { puntos gana y } \\
\text { termina el juego. }\end{array}$ \\
\hline \multicolumn{5}{|c|}{ Programa: La Máquina Del Millón (Del original hebreo “משאבתרכ" Bomba de dinero) } \\
\hline $\begin{array}{l}\text { Responder a ocho } \\
\text { preguntas para } \\
\text { detener la máquina } \\
\text { del millón y obtener } \\
\text { puntos. }\end{array}$ & $\begin{array}{l}04 \text { niveles o } \\
\text { secuencias }\end{array}$ & \begin{tabular}{|l|} 
Es un concurso de a \\
dos, los participantes \\
deberán responder \\
correctamente de \\
acuerdo a cada tema \\
de su preferencia.
\end{tabular} & $\begin{array}{l}\text { Al inicio la pareja tiene } \\
\text { un millón de soles } \\
\text { virtuales, monto que va } \\
\text { disminuyendo con el } \\
\text { cronometro en reversa } \\
\text { en cada pregunta. }\end{array}$ & $\begin{array}{l}\text { Si responden errado los } \\
\text { dos, o si se acaba el } \\
\text { dinero en la máquina, } \\
\text { termina el juego. }\end{array}$ \\
\hline
\end{tabular}

\section{Análisis de la prueba de entrada}

El experimento se inicia con el diseño y elaboración de diez (10) sesiones de aprendizaje referidas a: 1) La indumentaria; 2) El transporte; 3) Las profesiones; 4) La familia; 5) Las partes del cuerpo humano; 6) Los deportes; 7) Las nacionalidades; 8) Los objetos personales afines a la edad; 9) Verbos de actividades cotidianas; y 10) El deletreo (spelling), con el propósito de desarrollar las habilidades lingüísticas del idioma inglés. En este caso, se han considerado solo tres habilidades: hablar, escuchar, escribir, por tratarse del aprendizaje del vocabulario inglés, que exige conocer las palabras que una persona emplea en una primera instancia. La lectura y la comprensión de lectura en el idioma ingles será una actividad ulterior, por cuanto son habilidades que consisten en interpretar y descifrar el valor fónico de una serie de signos escritos. Por lo tanto, no siendo aún imprescindible comprender el contexto, se han evaluado tres habilidades lingüísticas: el dominio de la expresión oral, la comprensión auditiva y la expresión escrita.

\section{Análisis del nivel de aprendizaje del vocabulario inglés antes del experimento}

Aplicada la pre-prueba o pre-test, los resultados de la evaluación del aprendizaje del vocabulario inglés en las Instituciones Educativas IE1 y IE2 son presentados en la Tabla 3 con similares promedios. No se ha considerado la habilidad de comprensión lectora, como se ha explicado, siendo el objetivo mejorar en los estudiantes el aprendizaje de un conjunto de palabras de la lengua inglesa en primera instancia. 
Tabla 3: Evaluación Pre-Test de aprendizaje del vocabulario inglés

\begin{tabular}{|c|r|r|r|r|r|r|r|r|r|r|r|r|}
\hline \multirow{2}{*}{ NIVEL } & \multicolumn{4}{|c|}{ Pre-Test Listening } & \multicolumn{4}{|c|}{ Pre-Test Writing } & \multicolumn{2}{|c|}{ Pre-Test Speaking } \\
\cline { 2 - 13 } & \multicolumn{2}{|c|}{ IE1 } & \multicolumn{1}{|c|}{ IE2 } & \multicolumn{2}{|c|}{ IE1 } & \multicolumn{2}{|c|}{ IE2 } & \multicolumn{2}{|c|}{ IE1 } & \multicolumn{2}{|c|}{ IE2 } \\
\cline { 2 - 13 } & $\mathrm{F}$ & $\%$ & $\mathrm{~F}$ & $\%$ & $\mathrm{~F}$ & $\%$ & $\mathrm{~F}$ & $\%$ & $\mathrm{~F}$ & $\%$ & $\mathrm{~F}$ & $\%$ \\
\hline $\mathrm{AD}$ & 2 & $10 \%$ & 0 & $0 \%$ & 0 & $0 \%$ & 0 & $0 \%$ & 0 & $0 \%$ & 0 & $0 \%$ \\
\hline $\mathrm{A}$ & 0 & $0 \%$ & 0 & $0 \%$ & 1 & $5 \%$ & 0 & $0 \%$ & 0 & $0 \%$ & 0 & $0 \%$ \\
\hline $\mathrm{B}$ & 1 & $5 \%$ & 1 & $6 \%$ & 4 & $20 \%$ & 2 & $13 \%$ & 1 & $5 \%$ & 0 & $0 \%$ \\
\hline $\mathrm{C}$ & 17 & $85 \%$ & 15 & $94 \%$ & 15 & $75 \%$ & 14 & $88 \%$ & 19 & $95 \%$ & 16 & $100 \%$ \\
\hline TOTAL: & 20 & $100 \%$ & 16 & $100 \%$ & 20 & $100 \%$ & 16 & $100 \%$ & 20 & $100 \%$ & 16 & $100 \%$ \\
\hline
\end{tabular}

En la Fig. 1: El Pre-Test Listening, muestra en promedio que $89,5 \%$ de estudiantes necesitan refuerzo "C"; $5,5 \%$ está en proceso "B", y $5 \%$ alcanza nivel destacado "AD". En la Fig. 2 El Pre-Test Writing, muestra en promedio que $81,5 \%$ de estudiantes necesitan refuerzo "C"; $16,5 \%$ están en proceso "B", y $2,5 \%$ está en el nivel logrado "A". En la Fig. 3 Pre-Test Speaking, muestra en promedio que 97,5\% de estudiantes necesitan refuerzo "C", y $2,5 \%$ está en proceso "B".

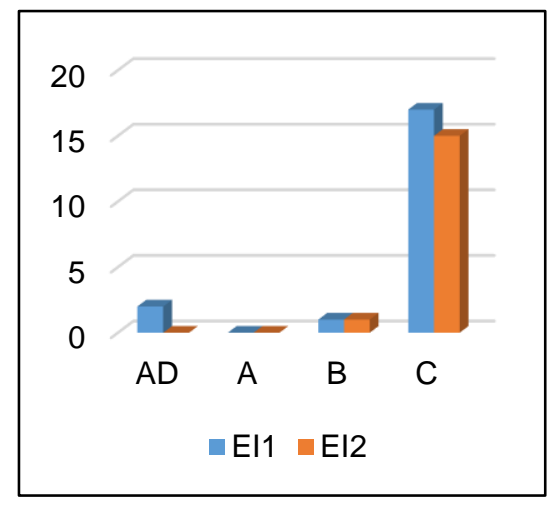

Fig. 1: Pre-Test Listening

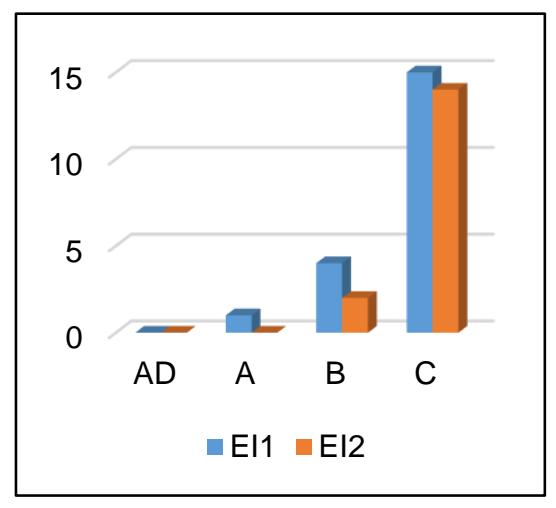

Fig. 2: Pre-Test Writing

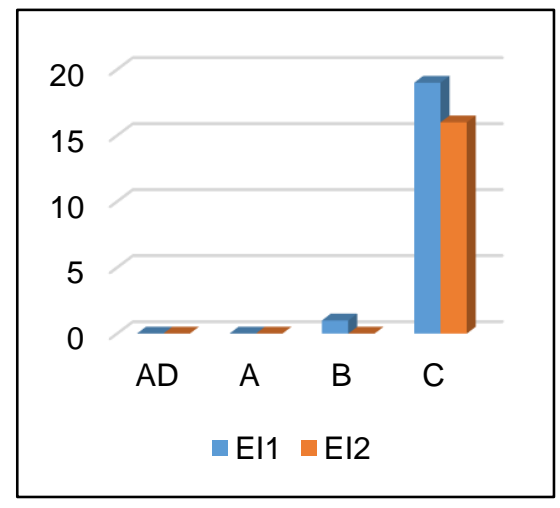

Fig. 3: Pre-Test Speaking

En resumen, el porcentaje de estudiantes con nota aprobatoria, entre destacado, logro y en proceso de 11 a 20 puntos es de $11,1 \%$ (Listening, Writing, Speaking).

\section{Aplicación experimental de la Técnica Didáctica JSPCT}

La Técnica Didáctica de Juego y Simulación de Programas Concurso de Televisión JSPCT proporciona un entorno diferente al aula tradicional, al aprovechar la televisión como medio de información, comunicación y entretenimiento, con actividades de enseñanza muy intensas, aprendizajes significativos con autonomía y altos niveles de motivación para explorar e investigar, desarrollando capacidades y competencias para el aprendizaje de otros idiomas, el trabajo en equipo y el aprendizaje cooperativo, interactivo y centrado en la resolución de problemas cotidianos de comunicación, en una lengua diferente a la materna.

\section{Los momentos de enseñanza aprendizaje}

La secuencia didáctica se sustenta en la taxonomía revisada de Benjamín Bloom (Churches, A. 2008), cuyas sub categorías tienen un número de verbos asociados a las habilidades de pensamiento en orden ascendente, desde las Habilidades de Pensamiento de Orden Inferior (LOTS) hasta las Habilidades de Pensamiento de Orden Superior (HOTS), considerados en la técnica didáctica JSPCT como momentos de enseñanza aprendizaje, tal como se puede observar en la Tabla 4, que no se restringen al ámbito cognitivo, sino que se apoyan en métodos y herramientas que permiten la calidad de la acción de la enseñanza y del aprendizaje, atendiendo nuevos comportamientos, nuevas acciones, nuevas técnicas y herramientas didácticas, y nuevas estrategias apoyadas en las TIC.

Se considera que la Taxonomía revisada de Bloom (RBT), es "una herramienta para ayudar a los educadores a aclarar y comunicar lo que pretendían que hicieran los estudiantes". Aprender como resultado de la instrucción "(Anderson y Krathwohl, 2001). Incorporado en el RBT son avances en la enseñanza y el aprendizaje desde la publicación del original. El término conocimiento era considerado un término inapropiado para describir una categoría de pensamiento y fue reemplazado por el término recordando. Además, la 
revisión reconceptualizó la taxonomía original de una sola dimensión en dos dimensiones con una Dimensión de Proceso Cognitivo y una Dimensión de Conocimiento, como dice Mary J. Pickard (2007), pág. 47.

Tabla 4: Momentos de enseñanza aprendizaje de la Técnica Didáctica JSPCT

\begin{tabular}{|c|c|c|}
\hline Momento & Actividad & Verbos \\
\hline Recordar & Muestra de varios flashcard. & $\begin{array}{l}\text { Reconocer, listar, describir, identificar, recuperar, } \\
\text { denominar, localizar, encontrar. }\end{array}$ \\
\hline Entender & $\begin{array}{l}\text { Se inicia generando conflicto cognitivo, para luego } \\
\text { pensar. }\end{array}$ & $\begin{array}{l}\text { Interpretar, resumir, inferir, parafrasear, clasificar, } \\
\text { comparar, explicar, ejemplificar. }\end{array}$ \\
\hline Aplicar & $\begin{array}{l}\text { Los estudiantes y el profesor organizan y ejecutan } \\
\text { el juego y la simulación. }\end{array}$ & Implementar, desempeñar, usar, ejecutar. \\
\hline Analizar & $\begin{array}{l}\text { El profesor reflexiona y pregunta en forma } \\
\text { consecutiva sobre cada una de las alternativas. }\end{array}$ & $\begin{array}{l}\text { Comparar, organizar, deconstruir, atribuir, } \\
\text { delinear, encontrar, estructurar, integrar. }\end{array}$ \\
\hline Evaluar & Aplica pre test y pos test. & $\begin{array}{l}\text { Revisar, formular hipótesis, criticar, experi- } \\
\text { mentar, juzgar, probar, detectar, monitorear }\end{array}$ \\
\hline Crear & $\begin{array}{l}\text { Los estudiantes escriben oraciones acerca de sus } \\
\text { rutinas diarias dando uso al IS, ARE, AM/ ISN'T, } \\
\text { AREN'T, AM NOT. }\end{array}$ & $\begin{array}{l}\text { Diseñar, construir, planear, producir, idear, trazar, } \\
\text { elaborar. }\end{array}$ \\
\hline
\end{tabular}

\section{Secuencia didáctica}

La secuencia didáctica con la estrategia didáctica JSPCT se presenta en la Tabla 5. Se incluye los recursos didácticos y el tiempo estimado para cada acción.

Tabla 5: Secuencia didáctica con la estrategia didáctica JSPCT

\begin{tabular}{|c|c|c|}
\hline Secuencia didáctica JSPCT (ejemplo) & Recursos didácticos & $t(\min )$. \\
\hline \multicolumn{3}{|l|}{ Saludo: "How Are You" } \\
\hline MOMENTO DE RECORDAR. & Flashcard de profesiones, de lugares geográficos, etc. & 5 \\
\hline MOMENTO DE ENTENDER. & $\begin{array}{l}\text { Pizarra y Plumones } \\
\text { Texto de inglés }\end{array}$ & 10 \\
\hline $\begin{array}{l}\text { MOMENTO DE APLICAR. } \\
\text { INICIO DEL JUEGO Y SIMULACIÓN }\end{array}$ & $\begin{array}{l}\text { Pizarra y Plumones } \\
\text { Video: } 100 \text { peruanos dicen } \\
\text { Proyector multimedia, Smartphone y Tablet }\end{array}$ & 40 \\
\hline MOMENTO DE ANALIZAR. & $\begin{array}{l}\text { Proyector multimedia } \\
\text { Pizarra y Plumones }\end{array}$ & 10 \\
\hline MOMENTO DE EVALUAR. & $\begin{array}{l}\text { Ficha de observación y Ficha de actitudes. } \\
\text { Prueba objetiva }\end{array}$ & 15 \\
\hline MOMENTO DE CREAR. & $\begin{array}{l}\text { Cuadernos y Lapiceros } \\
\text { Smartphone y Tablet }\end{array}$ & 10 \\
\hline
\end{tabular}

La secuencia didáctica corresponde a cada momento de la taxonomía revisada de Benjamín Bloom.

\section{Momento de Recordar}

Momento de hacer memoria, los conocimientos previos y la motivación. El profesor muestra flashcard de las profesiones, lugares geográficos, etc. luego pregunta, ¿Qué es lo que vieron en el flascard? ¿Cuáles son tus profesiones favoritas? ¿Qué lugares te gustaría conocer más? ¿En qué te gustaría trabajar cuando terminen la educación secundaria? ¿Cuáles profesiones son más riesgosas? ¿Cuáles profesiones son más lucrativas, loables?, etc.

\section{Momento de Entender}

Momento de percibir con claridad. Se inicia generando conflicto cognitivo, buscando el cambio conceptual que genera en los estudiantes una situación contradictoria, entre lo que saben y los nuevos conocimientos provocando un desequilibrio cognitivo que conduce a un nuevo conocimiento. El profesor pregunta, ¿Cómo saludar a un extranjero en inglés? ¿Cómo preguntarle de que nacionalidad es?, ¿Qué deporte le gusta? ¿En 
que trabaja o que ocupación tiene? etc. Los estudiantes responden hasta descubrir el sentido profundo de las palabras. El profesor escribe en la pizarra "The ocupation and places" con la ayuda de los estudiantes se realiza una lista de ocupaciones, firefighter, mechanic, dancer, doctor, nurse, teacher, police officer, chef, photographer, taxi driver, secretary; airport, hardboard, church, bus station, library, school. Is he a teacher?, Yes, he is / No, he isn't; he is teaching at school?, Yes, he is / No, he isn't. Se les pide que elaboren oraciones con las ocupaciones y lugares preferidos dando uso del presente simple y el presente continuo.

\section{Momento de Aplicar}

Momento de poner en práctica los procedimientos adecuados para conseguir un fin. Se explora en internet y se busca el video de los programas de concurso de televisión. Se elige uno y se muestra el video del quiz show "100 PERUANOS DICEN" para que los estudiantes conozcan sobre las secuencias y reglas del juego (solo como ejemplo). Los estudiantes y el profesor organizan la simulación del juego, para ello conforman 02 equipos de 04 integrantes, eligen a un integrante como líder y se reúnen antes de dar una respuesta, si se equivocan pierden su turno y pasa automáticamente el turno al otro equipo, de tal manera que el grupo que acumule más puntaje es el que gana el juego. Se planifican y organizan actividades referidas a: 1) La indumentaria; 2) El transporte; 3) Las profesiones; 4) La familia; 5) Las partes del cuerpo humano; 6) Los deportes; 7) Las nacionalidades; 8) Los objetos personales afines a la edad; 9) Verbos de actividades cotidianas; y 10) El deletreo (spelling). La Metodología del juego en el aula es la misma del programa concurso "Cien peruanos dicen" de la televisión. Ver en https://www.youtube.com/watch?v=atOcg zllac

Se inicia el juego y simulación. - El profesor actúa simulando ser el conductor del programa, se elige a un representante de cada grupo para determinar por sorteo qué grupo obtiene el control de la primera pregunta. El que da la respuesta más popular tiene el control de la pregunta, si ninguno de los jugadores da una respuesta válida, los siguientes integrantes participan hasta acertar (la simulación es totalmente en inglés). El equipo que controla las preguntas debe intentar responder a las que quedan en el tablero. Si el equipo comete un error se le marca una "X". Tres "X" determinan cambio de equipo, el otro equipo responde a esa pregunta y se queda con los puntos acumulados.

El formato del programa presenta los resultados de la encuesta a 100 personas, se colocan las 5 respuestas más populares que cada grupo tendrá que acertar, por ejemplo: ¿Qué profesión te gustaría estudiar? ¿Qué ocupaciones a nadie le gustaría hacer por más dinero que le paguen? ¿Qué lugares del mundo te gustaría conocer?, etc. Se colocan las respuestas y deben coincidir con el tablero virtual que esconde las 5 respuestas más populares de 100 peruanos, las cuales se van develando conforme cada integrante del grupo menciona una respuesta. Las cinco respuestas se develan de mayor a menor, es decir, de la respuesta más popular a la menos popular. Doble: En determinada pregunta se duplica la puntuación de cada respuesta. Triple: En determinada pregunta se triplica la puntuación de cada respuesta. Dinero rápido (en nuestro caso, vale por un examen oral): Dos participantes del mismo equipo tienen que responder a las mismas preguntas en 20 segundos, el que participa en segundo lugar no puede repetir las mismas respuestas del primero. La meta es llegar a 200 puntos para ganar el juego.

\section{Momento de Analizar}

Momento de examinar detalladamente una cosa, separando o considerando por separado sus partes, para conocer sus características o cualidades, o su estado, y extraer conclusiones. El professor pregunta: ¿Cuándo se usa el: IS, ARE, AM / ISN'T, AREN'T, AM NOT? ¿Qué diferencia hay entre HE IS A TEACHER / HE IS TEACHING AT SCHOOL? Preguntan en español e inglés, escriben en español e inglés, dialogan en español e inglés.

\section{Momento de Evaluar}

Momento de valorar en función de criterios respecto a un conjunto de normas, con la ficha de observación y el examen escrito como prueba objetiva, sobre las capacidades: 1) Expresión oral: Nombra e identifica las ocupaciones y lugares (Listening and Speaking); 2) Producción de textos: Redacta oraciones haciendo uso de "IS, ARE, AM/ ISN'T, AREN'T, AM NOT" (Writing); Actitudes: Reconoce los errores que puede presentar en el tema y consulta al profesor para resolverlo. Preguntan en español e inglés, escriben en inglés.

\section{Momento de Crear}

Momento de producir un texto en inglés. Los estudiantes escribirán en sus cuadernos una serie de oraciones usando: IS, ARE, AM / ISN'T, AREN'T, AM NOT. Preguntan en español e inglés, escriben en inglés. 


\section{Análisis del nivel de aprendizaje del vocabulario inglés después del experimento}

Concluida la aplicación experimental con la Técnica Didáctica JSPCT, se aplicó la prueba de salida con los resultados que se muestran en la Tabla 6, porcentaje de estudiantes con nota aprobatoria y desaprobatoria, como promedio de ambas instituciones educativas.

Tabla 6: Evaluación Post Test de aprendizaje del vocabulario inglés

\begin{tabular}{|c|c|c|c|c|c|c|c|c|c|c|c|c|}
\hline \multirow{3}{*}{ NIVEL } & \multicolumn{4}{|c|}{ Post -Test Listening } & \multicolumn{4}{|c|}{ Post-Test Writing } & \multicolumn{4}{|c|}{ Post -Test Speaking } \\
\hline & \multicolumn{2}{|c|}{ IE1 } & \multicolumn{2}{|c|}{ IE2 } & \multicolumn{2}{|c|}{ IE1 } & \multicolumn{2}{|c|}{ IE2 } & \multicolumn{2}{|c|}{ IE1 } & \multicolumn{2}{|c|}{ IE2 } \\
\hline & $F$ & $\%$ & $F$ & $\%$ & $F$ & $\%$ & $F$ & $\%$ & $F$ & $\%$ & $F$ & $\%$ \\
\hline$A D$ & 18 & $90 \%$ & 15 & $94 \%$ & 14 & $70 \%$ & 14 & $88 \%$ & 13 & $65 \%$ & 8 & $50 \%$ \\
\hline$A$ & 2 & $10 \%$ & 1 & $6 \%$ & 5 & $25 \%$ & 2 & $13 \%$ & 4 & $20 \%$ & 4 & $25 \%$ \\
\hline B & 0 & $0 \%$ & 0 & $0 \%$ & 0 & $0 \%$ & 0 & $0 \%$ & 3 & $15 \%$ & 4 & $25 \%$ \\
\hline C & 0 & $0 \%$ & 0 & $0 \%$ & 1 & $5 \%$ & 0 & $0 \%$ & 0 & $0 \%$ & 0 & $0 \%$ \\
\hline TOTAL: & 20 & $100 \%$ & 16 & $100 \%$ & 20 & $100 \%$ & 16 & $100 \%$ & 20 & $100 \%$ & 16 & $100 \%$ \\
\hline
\end{tabular}

En la Fig. 4, El Post Test Listening, muestra en promedio que 92\% de estudiantes lograron aprendizaje destacado "AD", y $8 \%$ aprendizaje logrado "B", ninguno en proceso o necesita refuerzo. En la Fig. 5, el Post Test Writing, muestra en promedio que $79 \%$ de estudiantes tiene aprendizaje destacado "AD"; $15 \%$ aprendizaje logrado "A" y solo un estudiante necesita refuerzo "C". En la Fig. 6, el Post Test Speaking, muestra en promedio que $57,5 \%$ de estudiantes alcanza aprendizaje destacado "AD"; $22,5 \%$ aprendizaje logrado "A", y $20 \%$ aprendizaje en proceso "B".

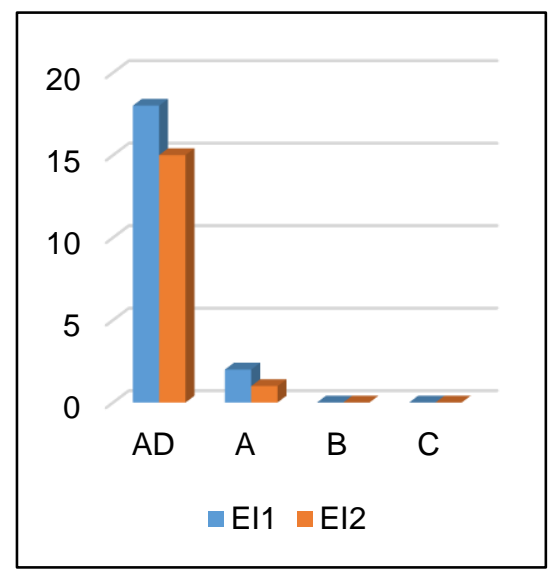

Fig. 4: Post Test Listening

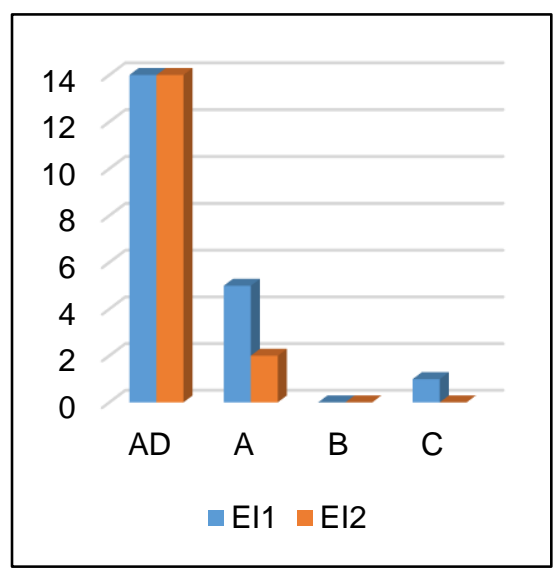

Fig. 5: Post Test Writing

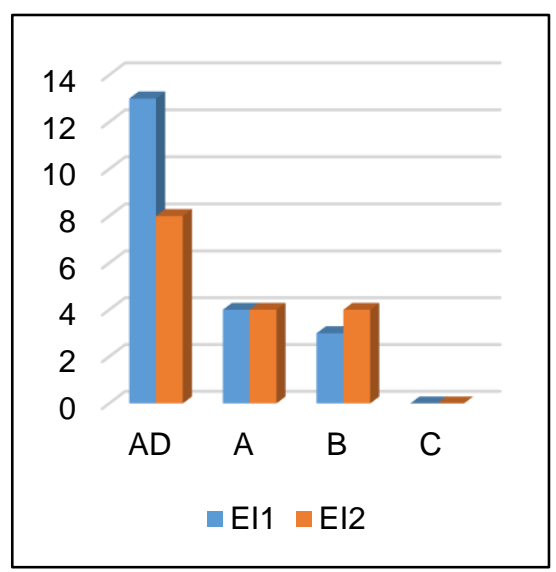

Fig. 6: Post Test Speaking

En resumen, el porcentaje de estudiantes con nota aprobatoria, entre destacado, logro y en proceso de 11 a 20 puntos es de $99,1 \%$.

\section{Discusión y Resultados}

El resultado más importante es que el proceso de enseñanza aprendizaje del vocabulario inglés ha mejorado notablemente. El porcentaje promedio de estudiantes con nota aprobatoria antes de la aplicación de la Técnica Didáctica JSPCT fue de 11,1\% y después de la aplicación es de 99,1\%, tal como se muestran en la Tabla 7 y Fig. 7. Esta enorme mejoría en los resultados del aprendizaje del vocabulario inglés en estudiantes de habla hispana se debe a la Técnica Didáctica JSPCT, al incrementar la participación de los estudiantes en el proceso de enseñanza aprendizaje, gamificando las actividades del aula, desarrollando habilidades de pensamiento, en los que es primordial la participación activa entre grupos pequeños, como indican $\mathrm{MP}$ Li, BH La, (2013), el aprendizaje cooperativo es una estrategia educativa facilitada por el instructor, centrada en el estudiante, que un pequeño grupo de estudiantes es responsable de su propio aprendizaje y el aprendizaje de todos los miembros del grupo. Los estudiantes interactúan entre sí en el mismo grupo para adquirir y practicar los elementos de un tema para resolver un problema, completar una tarea o lograr un Gol. Así como la comunicación constante, el juego y la diversión como elementos de motivación y creatividad, ante la intensa exposición de lengua oral y escrita, incrementan el sentido de competencia comunicativa, la precisión léxica 
y gramatical y las aplicaciones prácticas en la vida diaria, refuerzan la autoestima para comunicarse mejor con personas de habla inglesa.

La Técnica Didáctica JSPCT incita a los estudiantes a una acción determinada, cambiando su conducta para el aprendizaje; según Zolt’n Dörnyei (1998), la motivación ha sido ampliamente aceptada por los docentes y los investigadores como uno de los factores clave que influyen en la tasa y el éxito del aprendizaje de lenguas extranjeras (L2). La motivación proporciona el ímpetu primario para iniciar el aprendizaje de L2 y más tarde la fuerza motriz para sostener el largo y tedioso proceso de aprendizaje; de hecho, todos los demás factores implicados en la adquisición de L2 presuponen motivación en cierta medida. Sin una motivación suficiente, incluso las personas con las habilidades más notables no pueden lograr los objetivos a largo plazo, y tampoco los planes de estudios son apropiados y la buena enseñanza lo suficiente para garantizar el rendimiento del estudiante. Por otro lado, la alta motivación puede compensar deficiencias considerables tanto en la aptitud del lenguaje como en las condiciones de aprendizaje. En su trabajo seminal, Gardner y Lambert (1972) enfatizan que, aunque la aptitud del lenguaje representa una proporción considerable de la variabilidad individual en el logro del aprendizaje del lenguaje, los factores motivacionales pueden anular el efecto de la aptitud. En ciertos entornos idiomáticos, como señalan Gardner y Lambert, donde el entorno social lo exige (por ejemplo, cuando el LI es una lengua vernácula local y el L2 es el idioma nacional), muchas personas parecen dominar una L2, independientemente de sus diferencias de aptitud. Finalmente, la creatividad, considerada como la capacidad de generar nuevas ideas o conceptos, como potencialidades humanas que se construyen culturalmente desde los medios de comunicación como la televisión, permitirán un mejor aprendizaje de una segunda lengua, en programas que presentan juegos muy divertidos para niños, jóvenes y adultos, incluso con altos índices de sintonía.

Tabla 7: Resumen de las calificaciones antes y después de aplicar la Técnica Didáctica JSPCT

\begin{tabular}{|c|c|c|c|c|c|}
\hline Área: Inglés & \multirow{5}{*}{ 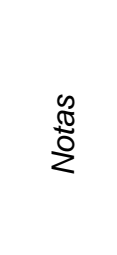 } & \multirow{3}{*}{\multicolumn{2}{|c|}{ Grupos IE1 e IE2 }} & \multirow{2}{*}{\multicolumn{2}{|c|}{ Grupos IE1 e IE2 }} \\
\hline Grupo $A=$ Arequipa & & & & & \\
\hline Grupo B = Juliaca & & & & & \\
\hline \multirow{2}{*}{ Niveles } & & \multicolumn{2}{|c|}{ Pre-test } & \multicolumn{2}{|c|}{ Post-test } \\
\hline & & $F$ & $\%$ & $F$ & $\%$ \\
\hline$A D=$ Destacado & 18 a 20 & 0 & $0.00 \%$ & 25 & $69.44 \%$ \\
\hline$A=$ Logro (bueno) & 15 a 17 & 0 & $0.00 \%$ & 10 & $27.78 \%$ \\
\hline $\mathrm{B}=$ En proceso (regular) & 11 a 14 & 3 & $8.33 \%$ & 1 & $2.78 \%$ \\
\hline $\mathrm{C}=$ Necesita refuerzo (bajo) & 0 a 10 & 33 & $91.67 \%$ & 0 & $0.00 \%$ \\
\hline Total: & & 36 & $100 \%$ & 36 & $100 \%$ \\
\hline
\end{tabular}

Específicamente y como se muestra en la Tabla 7 y en la Fig. 7, los estudiantes elevaron el nivel de aprendizaje del vocabulario inglés en el nivel Destacado "AD" de 0,0\% a 69,44\%; en el nivel de Logro " $A$ " de $0,0 \%$ a $27,78 \%$, disminuyendo el porcentaje en el nivel "B" de 8,33 a 2,78, y en el nivel "C" de 91,67\% a 0,0\%, lo que demuestra que la Técnica Didáctica JSPCT es recomendable para superar los problemas de enseñanza de lenguas extranjeras ELE y mejoran significativamente el nivel de aprendizaje de otros idiomas, en este caso del inglés en estudiantes de habla hispana.

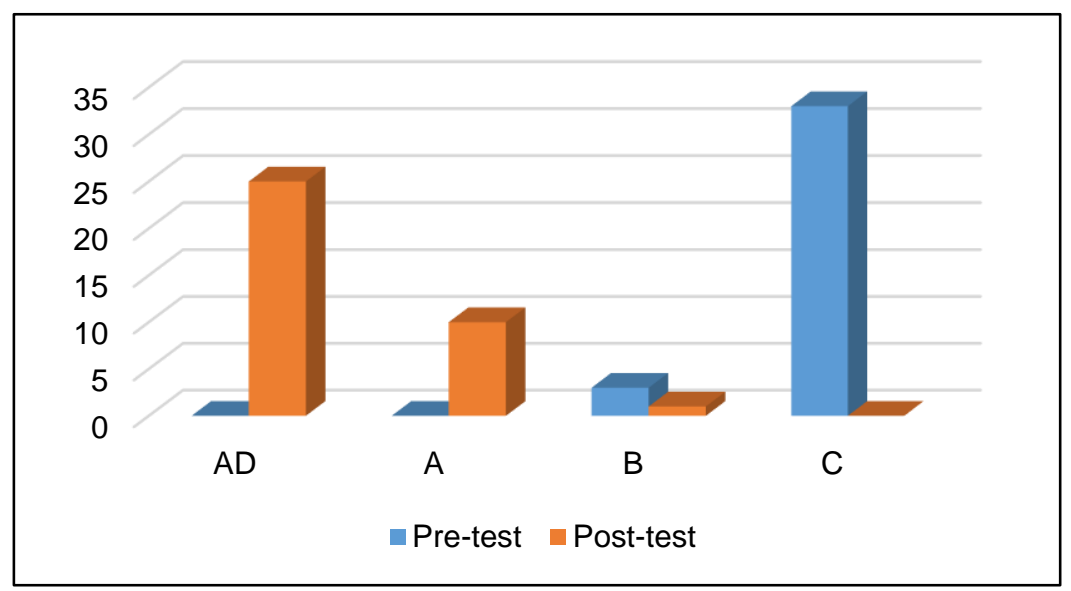

Fig. 7: Resumen de calificaciones Pre test y Post Test 
Los conceptos que sustentan la Técnica Didáctica JSPCT y otros resultados que respaldan el proceso, de enseñanza aprendizaje del vocabulario del idioma inglés, están referidos al uso de la televisión como medio masivo de comunicación, las TIC omnipresentes en toda actividad humana, la gamificación como concepto de aprendizaje lúdico y divertido y los problemas de aprendizaje del idioma inglés en estudiantes de habla hispana, como un esfuerzo constante de los profesores por superar la enseñanza - aprendizaje del idioma inglés, como señalan TM Connolly, M Stansfield, T Hainey (2011), en Juegos y aprendizaje de idiomas.- Como señaló Crookall (2007), los profesores de idiomas hacen un gran uso de las metodologías de simulación / juego y hay muchos apoyos, libros de texto y documentos de investigación que presentan diversas formas de juego de roles, juegos, simulaciones y otros ejercicios (por ejemplo, Garcia-Carbonell, Rising, Montero, y Watts, 2001; Gaudart, 1999; Halleck, 2007). Lógicamente entre ellas las TIC, según la American Academy of Pediatrics, 1995, citado en Rojas Osorio, V. (2008), en las últimas décadas, junto con el explosivo desarrollo tecnológico, especialmente de las llamadas Tecnologías de la Información y Comunicación (TIC) que incluyen televisión (TV), radio, música, videojuegos, telefonía, Internet, etc.; se ha comprobado también, la gran influencia que estas tecnologías, a través de su omni-presencia en los hogares, escuelas, instituciones, etc., tienen sobre la salud infanto-juvenil. Nuevas investigaciones, han dado cuenta de dicha influencia, particularmente en aspectos como conducta violenta, consumo de alcohol y drogas, conductas sexuales de riesgo, trastornos alimentarios, nutritivos y otros; o también la Canadian Paediatric Society, 2003, citado en Rojas Osorio, V. (2008), muchas de estas investigaciones han comenzado a dar luces sobre las bases neurológicas y neuropsicológicas de este fenómeno y las autoridades de salud de muchos países desarrollados han implementado políticas y medidas regulatorias y de educación para los niños y sus familias.

La Técnica Didáctica JSPCT tiene como base el juego y la simulación, en suma, una innovación de la gamificación, como un tipo de aprendizaje que transpone la mecánica de los juegos de televisión al proceso de enseñanza de una segunda lengua con el fin de conseguir altos niveles de aprendizaje, como una posible solución a las dificultades de la enseñanza - aprendizaje del idioma inglés como segunda lengua, por tratarse de una técnica didáctica altamente motivadora, cooperativa, horizontal, que imita situaciones reales, como indica WC Kriz (2003), sobre la simulación y el juego como método para modelar y cambiar situaciones; Los juegos de simulación representan modelos dinámicos de situaciones reales (una reconstrucción de una situación o realidad que es en sí misma una construcción social). Los juegos de simulación ayudan a imitar procesos, redes y estructuras de sistemas específicos existentes. Además, reflejan sistemas de la vida real, los juegos de simulación incorporan jugadores que asumen roles. El prototipo de simulación de juego combina juego de roles y simulación. Los juegos de simulación incluyen actores, reglas y recursos (Klabbers, 1999). En el campo de la educación, investigaciones llevadas a cabo en escuelas y universidades (véase informe NMC Horizon Report sobre Educación Superior, 2014) han señalado también el valor de la gamificación en los procesos de enseñanza y aprendizaje (Foncubierta, J.M., 2014). Entre todas sus bondades muchos estudios destacan el aumento de la implicación de los estudiantes en actividades que, a priori, podrían considerarse como aburridas o difíciles, produciéndose así una mayor motivación del sujeto que aprende; un estímulo positivo procedente, en muchas ocasiones, del reconocimiento al esfuerzo, el premio y los sistemas de retroalimentación que permite la sensación de juego.

\section{CONCLUSIONES}

De los resultados mostrados, del análisis y discusión, se pueden obtener las siguientes conclusiones sobre la aplicación de la Técnica Didáctica JSPCT: 1) La característica principal de la metodología empleada radica en la mejora significativa del aprendizaje del vocabulario inglés en estudiantes de habla hispana; 2) La técnica propuesta es altamente motivadora al utilizar el juego y la simulación, gamificando el proceso de enseñanza aprendizaje con el apoyo de programas concurso de la televisión; 3) Las diferencias del nivel de aprendizaje del vocabulario inglés como segunda lengua son enormes, se ha invertido el porcentaje de logro de los aprendizajes del vocabulario inglés, en el nivel Destacado "AD" de 0,0\% a 69,44\%, en el nivel de Logro "A" de $0,0 \%$ a $27,78 \%$, y disminuyó el porcentaje en el nivel En proceso "B" de 8,33 a 2,78, y en el nivel Necesita refuerzo "C" de 91,67\% a 0,0\%; 4) La Técnica Didáctica de Juego y Simulación de Programas Concurso de Televisión JSPCT proporciona un entorno diferente al aula tradicional, al aprovechar la televisión como medio de información, comunicación y entretenimiento, con actividades de enseñanza muy intensas y con aprendizajes altamente significativos.

\section{AGRADECIMIENTOS}

A la U. Nacional de San Agustín de Arequipa, por el financiamiento de la investigación: Los Juegos de Televisión (programas de concursos de conocimiento, game shows) y el Aprendizaje del Vocabulario inglés en los estudiantes del Cuarto Grado de Educación Secundaria de las Instituciones Educativas Particulares Daniel Goleman de Juliaca y Jens Knudsen de Arequipa, ganadora de una subvención, contrato N 97-2016UNSA en la primera convocatoria, "Tesis para optar el título profesional", que da origen al presente artículo, en el que participó el equipo de investigación, a la convocatoria del convenio de Cienciactiva-UNSA de CONCYTEC Perú, al haber monitoreado y evaluado la investigación inicial. 


\section{REFERENCIAS}

American Academy of Pediatrics, Committee on Communications: Children, adolescents, and television, Pediatrics, 96, 786-7 (1995)

Andreu, A.A., C.M. García y G.M. Mollar, La Simulación y Juego en la Enseñanza-Aprendizaje de Lengua Extranjera, Cuadernos Cervantes, ISSN: 11349468, XI (55), 34-38 (2005)

Canadian Paediatric Society, Psychosocial Paediatrics Committee, Impact of media use on children and youth, Paediatrics \& Child Health, 8, 301-6 (2003)

Connolly, T.M., M. Stansfield y T. Hainey, An alternate reality game for language learning, ARGuing for multilingual motivation - Computers \& Education (2011)

Churches, A., Welcome to the $21^{\text {st }}$ Century (2008)

Foncubierta, J.M., Gamificación y aprendizaje de segundas lenguas, Edinumen, Madrid (2014)

Foncubierta, J.M., La simulación y juego en la enseñanza-aprendizaje de lengua extranjera (2015)

Huapaya C.R., G.M. Arona y F.A. Lizarralde, Enseñanza de la Ingeniería con Sistemas Tutoriales Inteligentes, Información Tecnológica, 16(5), 75-78 (2005)

Kriz, W.C., Creating effective learning environments and learning organizations through gaming simulation design Simulation \& Gaming, 2003 - journals.sagepub.com (2003)

Li, M.P. y BH. Lam, Cooperative learning, The Active Classroom, The Hong Kong Institute of ..., 2013 - eduhk.hk (2013)

Lorente F.P. y C.M. Pizarro, El Juego en la Enseñanza de Español como Lengua Extranjera, Nuevas Perspectivas, Revista de Estudios Filológicos, № 23 Julio (2012)

Marías, J., Ni bilingüe ni enseñanza, El País Semanal (2015)

Mary J.P., Journal of Family and Consumer Sciences Education, Vol. 25, No. 1, Spring / Summer, p. 47, The New Bloom's Taxonomy: An Overview for Family and Consumer Sciences, East Carolina University (2007)

Moeller, A., Kramer y T. Catalano, Foreign Language Teaching and Learning, Faculty Publications, Department of Teaching, Learning and Teacher Education (2015)

Oster, U., La adquisición de vocabulario en una lengua extranjera: De la teoría a la aplicación didáctica, Porta Linguarum, ISSN: 1697-7467, (11), 33-50 (2009)

Rojas Osorio, V., Influencia de la televisión y videojuegos en el aprendizaje y conducta infanto-juvenil, Revista Chilena de Pediatría, Vol. 79, Suplemento 1, (2008)

Ruiz M.R. y E.G.M. García, Las estrategias de aprendizaje y sus particularidades en lenguas extranjeras, Revista Iberoamericana de Educación, 36(4), 8 (2005)

Sarason, Y. y C. Banbury, Active learning facilitated by using a game-show format or who doesn't want to be a millionaire? DOI: 10.1177/1052562903260808, Journal of Management Education, 28 (4) 509-518 (2004)

Valderrama, J.O., Asuntos Éticamente Reprochables en una Publicación Internacional, V Encuentro de Editores de Revistas Científicas, Instituto de Ciencia Animal, La Habana-Cuba, Noviembre (2004)

Yassaei, S., Using Original Video and Sound Effects to Teach English. In English teaching forum (Vol. 50, No. 1, pp. 1216). US Department of State. Bureau of Educational and Cultural Affairs, Office of English Language Programs, SA-5, 2200 C Street NW 4th Floor, Washington, D.C. 20037 (2012)

Zolt'n, D., Motivation in second and foreign language learning, DOI:10.1017/S026144480001315X, Language Teaching/Volume 31/Issue 03/July 1998, 117-135 (1998) 